\title{
An Environmental Management Plan in Vavouto harbor (New Caledonia) with a statistical treatment displayed on dynamic maps
}

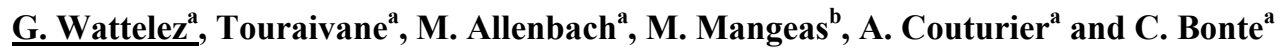 \\ ${ }^{a}$ University of New Caledonia, ${ }^{b}$ Institut de Recherche pour le Développement \\ Email: guillaume.wattelez@univ-nc.nc
}

\begin{abstract}
This paper presents an environmental monitoring tool to control and share results in real time as part of an environmental management plan (EMP) associated with a major dredging operation in the northern part of the New Caledonian lagoon. The interoperable tool includes results collected by a network of sensors that monitor turbidity and other physicochemical parameters of both the water and sediment.

The tool was designed for the spatial and temporal monitoring of turbidity using interpolation maps at daily, weekly, monthly, and annual intervals.

The geostatistical method of kriging was chosen for interpolation of turbidity values from the network of sensors in Vavouto harbor. Several maps are produced that show turbidity values based on whether they are one-off on-site measurements or measurements recorded continuously by sensors with radio or manual transmission, along with the depth of the measurement.

The Open Geospatial Consortium (OGC) provides the framework for collecting, displaying, processing, and sharing results on dynamic maps to comply with international standards. These standards were used to produce a dynamic map, and water quality data from sensors installed in the lagoon near Vavouto were stored and shared using Observations and Measurements (O\&M) and Sensor Observation Service (SOS) standards. This map, which provides results in real time, is accessible on the web.

The creation of a standardized tool for the acquisition and organization of data based on mathematical data processing methods enables an integrated approach to environmental management planning, and can be shared by all stakeholders.
\end{abstract}

Keywords: Environmental management plan, water quality in the lagoon, interoperable tool, statistical treatment in real time, Open Geospatial Consortium standards 


\section{INTRODUCTION}

The nickel mining project in the Koniambo massif raises high hopes for achieving a balance between economic and social development of New Caledonia. This major project includes the construction of an ore processing plant and of a port in Vavouto lagoon, North Province. A $4500 \mathrm{~m}$ long, $120 \mathrm{~m}$ wide, $12 \mathrm{~m}$ deep navigation channel that will require moving 7.3 million $\mathrm{m}^{3}$ of material is needed for the construction of the metallurgical complex and subsequently for the export of finished products (Touraïvane et al., 2011). Dredging is indispensable for the completion of this world scale project, and will affect the "VKP area", an acronym for the three municipalities involved: Voh, Koné, and Pouembout.

Dredging will be performed in the New Caledonian lagoon, close to areas classified as UNESCO World Heritage Sites for their exceptional biodiversity (Allenbach et al., 2010). An environmental management plan (EMP) was required by INERIS (Intitut National de l'Environnement Industriel et des Risques http://www.ineris.fr/) in 2005, to monitor the impact of the works on this delicate environment, especially because close links to the natural environment are part of the culture of the indigenous people in New Caledonia.

First we give a brief description of environmental monitoring, followed by a more detailed description of the user-friendly tool that was built to enable temporal and spatial monitoring of turbidity using dynamic maps that can interpolate this physicochemical parameter. Results are displayed in real time. We explain how the tool was made interoperable and discuss the advantages of interoperability.

\section{ENVIRONMENTAL MANAGEMENT PLAN FOR DREDGING VAVOUTO HARBOR}

The environmental management plan (EMP) specifies six issues to be monitored: water and air quality, ecology (flora and fauna), noise, waste management and hazardous substances. Of the six, our tool is only concerned with water quality, which is assessed through measurements of turbidity.

Turbidity is caused by the presence of suspended particles of clay, silt, sand, organic matter, plankton and other microorganisms in water. A system of fixed buoys was set up in the affected area to measure turbidity caused by dredging along with certain other parameters including water temperature, and the speed and direction of the wind.

Many data sets have been made available regarding turbidity: satellite images of the affected area, monthly activity reports of boats, etc. Unfortunately this mass of data was disorganized, so a conceptual model was first built based on the available data, followed by a physical data model that helped build a Postgres database, called the Vavouto database. The structure of the database allowed spatial analysis, assessment of the impacts of dredging, automatic updates of the database, and monitoring of turbidity.

The first version of tool was implemented in $R$ language ( $R$ Development Core Team, 2011). Its goal was to provide a temporal and spatial overview of turbidity in the form of maps showing interpolated data. The method of spatial interpolation chosen was kriging, as it helps understand the spatial structure of the phenomenon concerned. This method is based on the analysis of the covariance between sites of measurement. The analysis consists in choosing a variogram adapted with measurements. Various types of functions can be chosen. Functions of exponential type (Waller et al., 2004) are used in our tool and the variogram parameters (the reached level and the distance for which the level is reached) are computed thanks to an error minimisation between the theory variogram (exponential type) and the experimental variogram. Then a problem of optimization is resolved to assess the value of a variable (turbidity) at each point of the area of interest. This problem consists in minimizing the variance of the error between the theory value and the estimate while the estimate is without bias, i.e. the theory average and the estimate average are equal. This method has the advantage that the variance of the kriging estimates can be evaluated at any point in the area. It is then easy to determine the accuracy of the estimate provided by the interpolation at each point.

For a given day, the number of points on which measurements were taken is not constant. But it is necessary to have at least six points for the kriging to proceed. If there are less than six measurement points for one day, all depths being considered, the measurements of the closest days are taken in account in order to have a reliable interpolation. When the number of stations of measurements is sufficient for a day at a specific depth, a map showing the results for this depth is produced.

Several maps showing the results of the interpolation of the turbidity measurements were produced. Each map is a picture in which different levels of colour represent the different levels of turbidity. The map was available as an R script and the user could look at all the generated maps to check for a possible anomaly at any point of interest. We now offer this tool as a web service. 


\section{USING A WEB SERVICE FOR THE EMP OF DREDGING OPERATIONS AT VAVOUTO}

Making this tool available as a web service gave us the opportunity to make it more user-friendly. One way to achieve this objective is to present results on a dynamic map. Interoperability is also a useful feature, because the tool will be able to work with future systems without restricted access or implementation. Our tool thus complies with the Open Geospatial Consortium (OGC) specifications.

\subsection{OGC standards}

OGC provides some standards for storing, sharing and displaying geographic data. Table 1 shows such standards we used to build the web service for the EMP.

Other OGC standards such as WFS (Web Feature Service: for querying geographic features), WCS (Web Coverage Service: for extracting layers, i.e. geospatial information representing phenomena that vary spatially and temporally) and SAS (Sensor Alert Service: for notifying events), are available but not yet used for our tool. The use of these standards provides a structure for the organization of field data, regardless of the type of sensor used, observations made, or data collected. Once stored in a database with a consistent structure that complies with specified standards, the data are available and editable through a web service.
Table 1. OGC standards used in the tool built for the dredging monitoring at Vavouto

\begin{tabular}{|l|l|l|}
\hline Acronym & Meaning & Use \\
\hline GML & $\begin{array}{l}\text { Geography } \\
\text { Markup } \\
\text { Language }\end{array}$ & Describing geographic features \\
\hline WMS & $\begin{array}{l}\text { Web Map } \\
\text { Service }\end{array}$ & $\begin{array}{l}\text { Distributing geo-referenced } \\
\text { map images from a map server } \\
\text { using data from a GIS } \\
\text { (Geographic Information } \\
\text { System) database }\end{array}$ \\
\hline SensorML & $\begin{array}{l}\text { Sensor Model } \\
\text { Language }\end{array}$ & $\begin{array}{l}\text { Describing methods in sensor } \\
\text { systems and treatments }\end{array}$ \\
\hline O\&M & $\begin{array}{l}\text { Observations } \\
\text { and } \\
\text { Measurements }\end{array}$ & $\begin{array}{l}\text { Encoding observations or } \\
\text { geographic features involved } \\
\text { during observations }\end{array}$ \\
\hline SOS & $\begin{array}{l}\text { Sensor } \\
\text { Observation } \\
\text { Service }\end{array}$ & $\begin{array}{l}\text { Archiving sensor data available } \\
\text { on the web }\end{array}$ \\
\hline
\end{tabular}

\subsection{Structure of the tool: scientific calculations as a user action}

To be accessible on the web, the tool has to be hosted on a remote server. Every component of the tool is thus on a server, but some components can be on different servers. Below we explain which components can be separate. Table 2 summarizes software and concept components of the tool.

Data from sensors are stored in a PostgresSQL (http://www.postgresql.org/) database. The primary structure of the database was modified to comply with O\&M and SOS standards. The web-based SOS application of $52^{\circ}$ North (http://52north.org/) was found to be suitable for implementing an SOS service (Nüst et al., 2011) that provides data about dredging in Vavouto.

The R software was used for kriging. The calculation software is an important component of our tool as it has to be able to query the SOS database, process data, and provide outputs. Fortunately, R contains many libraries that make this possible. The sos4R library (Nüst, 2010) allows communication between $\mathrm{R}$ and an SOS service (Nüst et al., 2011). The sp library (Pebesma, 2004) provides a framework to handle spatial objects: spatial matrices, points, lines and polygons, and there is a function in the rgdal library (Keitt et al., 2012) to export R spatial objects to GML files.

A server-side programming language is required to undertake all the necessary actions (to collect the parameters sent by the user, to run the calculations, etc.) by the tool hosted on a remote server. We chose PHP (Hypertext Pre-Processor - http://php.net/). When a user sends a request, a PHP script takes care of running the corresponding $\mathrm{R}$ script with the parameters sent with the request.

Users visit a web page on the server, specify the parameters and start the calculations. AJAX (Asynchronous JavaScript And XML) is used to avoid reloading the web page. The user sends requests to the server, in our case, the parameters required for the execution of a script, which then processes them. Once the treatments complete, the server sends back a response that will change some parts of the web page from which the user request was sent. Our use of this concept is possible thanks to the JavaScript library: jQuery (http://jquery.com/).

Parameters are specified by filling in a form. The parameters are then sent to the remote server by a jQuery method (jQuery.ajax). On the server, a PHP script reads the user's settings. This script will also launch the R 
software installed on the server by specifying which $\mathrm{R}$ script to use ${ }^{1}$ with the settings sent by the user. The $\mathrm{R}$ software receives parameters, sends requests to the SOS to retrieve the needed information and performs the calculations. It creates several files that can be text files, tables, etc. Once the R script is fully executed, the PHP script takes over and reads the output files written by $\mathrm{R}$ to send the information they contain to the user so they can be displayed in the user's browser, or be downloaded. The stream sent by the server allows the page viewed by the user to be changed using JavaScript.

Table 2. Software and concepts used in the tool for the dredging monitoring at Vavouto

\begin{tabular}{|l|l|l|}
\hline Software / Concept & Category & Function \\
\hline PostgreSQL & $\begin{array}{l}\text { Database } \\
\text { management system }\end{array}$ & Stores and returns the sensor data \\
\hline SOS of $52^{\circ}$ North & Web service & Gathers and returns sensor data \\
\hline R & Processing software & Gathers data from the SOS, processes this data and creates outputs (GML files) \\
\hline PHP & $\begin{array}{l}\text { Programming } \\
\text { language }\end{array}$ & $\begin{array}{l}\text { Collects parameters from the user and gives them to R when it starts R processes } \\
\text { When R processes are ended, the PHP script gives output addresses to the user }\end{array}$ \\
\hline AJAX & Concept & Helps during exchanges between the user and the server \\
\hline jQuery & JavaScript library & Contains a method to use the AJAX concept \\
\hline OpenLayers & JavaScript library & Displays dynamic maps and overlays on a web page \\
\hline
\end{tabular}

\subsection{Structure of the tool: displaying dynamic maps with treatment outputs}

Dynamic maps are displayed thanks to OpenLayers (http://openlayers.org/). A WMS map server is queried and sends back the geo-referenced pictures that are embedded in the dynamic map.

When the results become available, they are automatically displayed on the dynamic map. The $\mathrm{R}$ software provides GML text outputs. These outputs describe the results of the statistical treatment requested by the user and are interpreted with OpenLayers for display on the map. An additional file created by R provides the address of the output file so that OpenLayers knows where to find it. As a result, the tool provides a dynamic map on which turbidity levels are exposed thanks to GML data.

\subsection{Tool overview}

Figure 1 shows the processes of the tool. A map of the area, obtained by a WMS request (1), and a form where the input parameters can be entered are then displayed. On the client side, the user sends the parameters to the server (2) which, in our case, controls the process execution (3), consisting in data recovery (4) and kriging (5) of the data. Files containing results are created (6) along with additional files describing the location of the GML output files (7). The server sends back a response including the addresses of the results file (8), which allows OpenLayers to display the content of the GML files as maps on the client side (9). All the files created during this all process are automatically destroyed once the user has quit the web page.

\section{DISCUSSION}

\subsection{Accuracy of the kriging interpolation}

An advantage of the kriging method is the ability to estimate the variance at each point of the area of interest and so to have an idea of the uncertainty linked with the interpolation. Even if the variance estimate is automatically computed during the R process, we chose to not display the variance map on our tool because we considered the function of the tool was mainly to provide an estimate of the turbidity level. Though it is not specified anywhere, the user must be aware that in general, the more distant is a point from a station of measurement, the less accurate is the interpolation. The kriging method used in this tool is the ordinary kriging with an isotropic variogram. The model could be more complex in order to expect more accurate

\footnotetext{
${ }^{1}$ Numerous R scripts are used in the tool. For instance the form with which the user selects the date is dynamically displayed after database queries in order to know which days are available. Database queries are done with $\mathrm{R}$ scripts. Then the statistical treatment is done with another $\mathrm{R}$ script.
} 
results but the processing time would be longer. Indeed, the expert (main user) would just like to know anomalies. As a result, an approximate estimate is sufficient in this case.

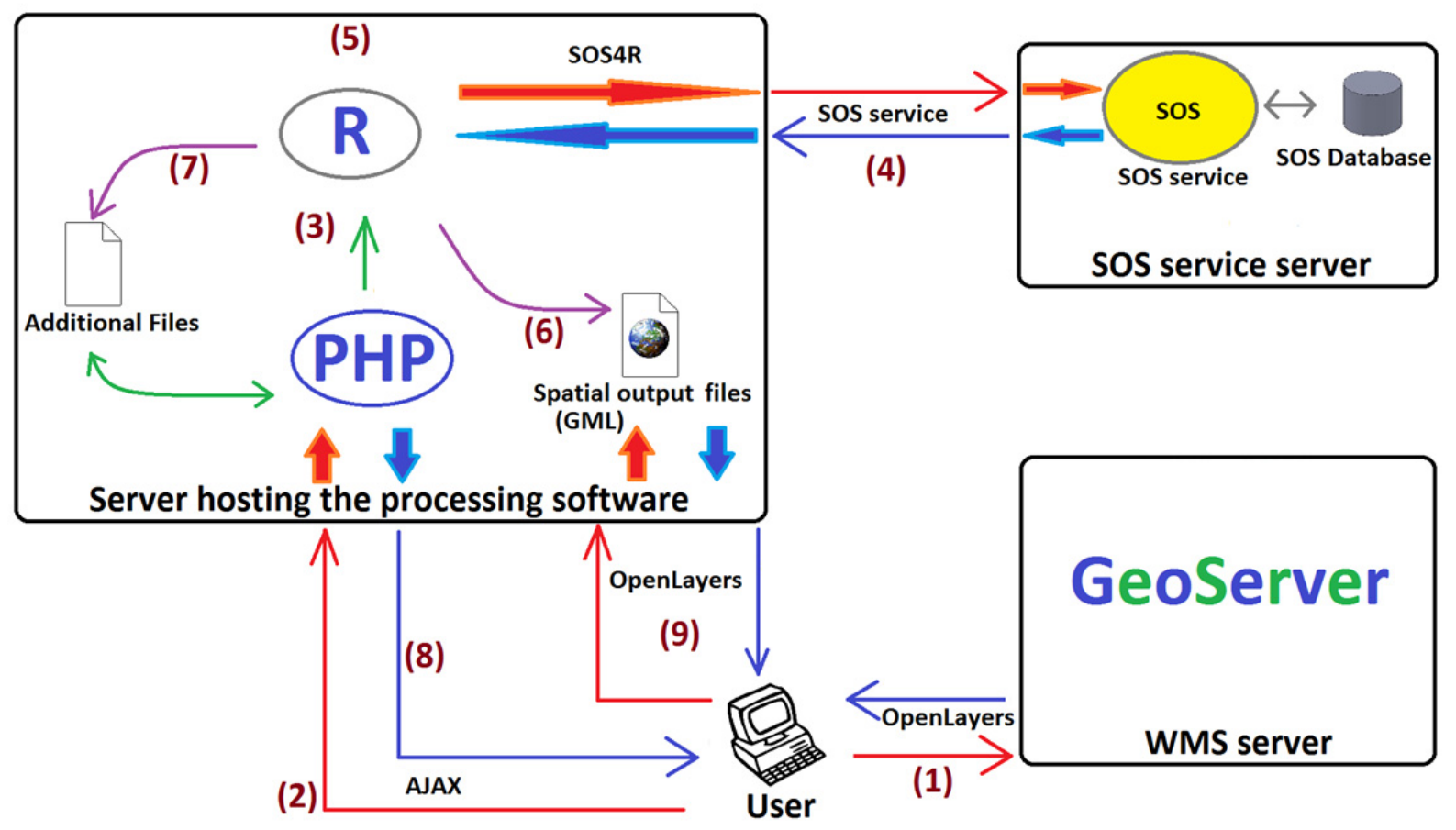

Figure 1. Interactions between technologies to allow the display of spatial results on a dynamic map in real time

\subsection{Advantages}

\section{Web service and dynamic maps}

As mentioned in section 0 above, a tool was previously available but it came as an $\mathrm{R}$ script the user had to run to obtain pictures of the Vavouto bay area with the turbidity assessment. To use this tool, it was necessary to install PostgreSQL, the Vavouto database, R, plus the packages needed to implement the tool.

Now that we propose this tool as a web service, users only need a web browser and an Internet connection (common). Users do not have to handle the processing software with which the tool was developed. Indeed, this step can be time consuming particularly if the tool is only rarely used: the "plasticity" of the human brain makes it very easy to forget mechanisms or concepts that are rarely used, even when we would prefer to remember them. In addition, if a new stakeholder becomes involved in the project, he/she has immediate access to the tool.

In our approach, the tool is easier to execute than a script. Finally, the use of dynamic maps helps to better understand the spatial and environmental context of the study site, by simply navigating and focusing on any area of interest.

From the designer's point of view, this approach also has many advantages, including centralization of the application. If an improvement is needed, it only has to be made where the tool is hosted. If necessary, a simple message can be inserted in the tool to inform users. In other words, the designer does not have to inform each user about a new version of the tool to be downloaded from a depository.

\section{OGC Standards}

The use of OGC standards helps make our tool interoperable. Interoperability makes it possible to access geographic entities published anywhere in the world on our dynamic map, provided the data managers allow access. Thus, if data, such as satellite images, are stored somewhere, are available and viewable on our map, it is possible to compare these additional external data and the kriging results for our own study area. Or 
maybe the introduction of a new variable that is not available with our tool, but is available elsewhere, could help explain an observed phenomenon or to find solutions to a problem encountered.

Using standards that apply to data collection and transmission from sensors is also very practical. We refer to the difficulties faced by expert analysts who want to exploit what is basically a mass of disorganized data (Touraïvane et al., 2011). The O\&M and SOS standards provide a clearly-defined framework for the data and methods to transmit or share data, so that the service that supplies data is interoperable.

It is quite possible that to be effective, an application requires information from several research teams. If each team's method of collecting and sharing data comply with OGC specifications, acquiring the information is facilitated. Even if the data is stored all over the world, the query form remains the same. Only the addresses of the SOS services differ. There is no need for clarification between teams about the structure of the database before sending a request for data collection or metadata. Connecting information from different teams is greatly facilitated. Standards then become a way of facilitating inter-team and interdisciplinary exchanges and work.

The implementation of the tool and the diffusion of results to stakeholders mean an integrated approach to environmental management is possible.

\subsection{Possible improvements}

\section{Monitoring in real-time}

For the moment, the data we use with this tool are past data. The tool could be improved by automatic filling of the database if not in real time, at least daily. This would greatly improve the EMP proceedings. In particular, if an expert identifies an anomaly in water quality, available tools allow him/her to combine all kinds of representations including climatic or environmental parameters, and with his/her expert point of view of the situation, he/she can deduce the cause of the anomaly. For example, in the case of an error in measurements made by a sensor, it would be useful to be able to rapidly correct the calibration of the faulty instrument; if the anomaly is due to works underway in the area, the expert can recommend that the works be halted or their intensity reduced; if the anomaly is caused by weather conditions, he/she will certainly make an appropriate decision to minimize the impact on the affected area, depending on the context.

Automatic filling of the database would enhance EMP implementation since monitoring could be almost in real time (taking into account the time needed to transmit the data and fill the database). Our tool is designed to support such an improvement. In addition, the O\&M and SOS standards help establish a system of automatic database filling, with a very simple query sent to an SOS web service.

\section{Other OGC standards}

Other OGC standards are applicable to our tool. For instance, the WPS (Web Processing Service) standard (http://www.opengeospatial.org/standards/wps) helps make processing interoperable, especially in the geographic information field (Open Geospatial Consortium Inc., 2007). It specifies how to request available data processing, metadata about the treatments (description of the inputs and outputs), and their execution. It also specifies how to respond to these requests. The three operations responsible for these exchanges are respectively: GetCapabilities, DescribeProcess and Execute. Exchanges between the client and the service are essentially done with XML and KVP flows.

Data processing can be simple or complex. It can be "generic", i.e. using data from anywhere; or "thematic", i.e. specific processing of specific data in a specific scientific field in a specific geographic area. For instance, our tool provides a thematic treatment: turbidity kriging in the Vavouto bay (the user only needs to select a date and a variable). But we could provide a treatment simply called "kriging" with which a user provides geographically referenced points and parameters for each point of interest with the aim of kriging in the area chosen by the user.

Finally, a warning system that is triggered when a threshold is exceeded would be extremely useful. If experts only need to know when a threshold has been exceeded so they can take an appropriate decision or action, a warning system would tell them exactly what kind of anomaly has been detected. In the case of an alert, the tool described here could be used. It would provide details such as the day the anomaly was detected thereby helping experts to take appropriate decisions based on his/her own observations. The SAS of OGC was created for this purpose. 


\section{CONCLUSION}

In this paper, we presented a tool for monitoring the Vavouto harbor area as part of the environmental management plan for dredging a navigation channel. This interoperable tool is available as a web service on the Internet; it helps display results of a statistical treatment thanks to dynamic maps that can be modified in real time. Dynamic maps are particularly useful for this type of study because they enable interactions with users who may wish to focus on a specific area or have a general view of the whole affected area, depending on their objectives. The tool is easy to use for stakeholders, who only need an Internet connection to be able to use it to full advantage. This facilitates interactions between different stakeholders who do not all need to have the same tool on their own computers.

OGC standards provide a framework for the collection, display, processing, and sharing of geographic data (standards such as WMS, WFS and WCS) on dynamic maps. Other standards related to sensor data (O\&M and SOS) are appropriate for archiving and sharing. The use of standards makes our tool interoperable. It is also possible to display additional data, perhaps the results of other teams from other services who also use OGC standards to help explain some events. Other standards for geographic data and processing provided by OGC would also be applicable to our tool.

The efficiency of our new tool will be further enhanced if new data are regularly collected and used to fill our database, possibly in real time. It is amenable for such an improvement, especially as the O\&M and SOS standards simplify the addition and deletion of data. An improvement of this type would give experts an overview of the situation in real time, allowing appropriate action to be taken rapidly depending on the situation. Combining our tool with an integrated warning system would improve it even more.

A tool of this type for use with environmental management plans would encourage both an integrated approach and the sharing of data between different stakeholders. In turn, this would improve both the efficiency and appropriateness of monitoring and encourage more reactive approaches to the conservation of World Heritage Sites and biodiversity.

\section{REFERENCES}

Allenbach, M., M. Mangeas, and Touraïvane (2010). Le suivi environnemental du dragage de Vavouto (Nouvelle-Calédonie). Un chantier de dimension mondiale à la porte d'un site classé au patrimoine mondial. XIèmes Journées Nationales Génie Côtier - Génie Civil. Les Sables d'Olonne: Paralia CFL, 2010, 573 - 582.

Keitt, T.H., R. Bivand, E. Pebesma, and B. Rowlingson (2012). rgdal: Bindings for the Geospatial Data Abstraction Library. http://CRAN.R-project.org/package =rgdal.

Nüst, D., C. Stasch, and E.J. Pebesma (2011). Connecting R to the Sensor. Advancing Geoinformation Science for a Changing World, Springer Lecture Notes in Geoinformation and Cartography. W. Reinhardt and F. (Eds.) Toppen, 227 - 246.

Nüst, D. (2010). sos4R: An R client for the OGC Sensor Observation Service. http://www.nordholmen.net/sos $4 r /$.

Open Geospatial Consortium Inc. (2007). OpenGIS Web Processing Service. Peter Schut, 8 June 2007.

Pebesma, E.J. (2004). Multivariable geostatistics in S: the gstat package. Computers \& Geosciences, 30, 683691.

R Development Core Team (2011). R: A language and environment for. Vienne: R Foundation for Statistical Computing, Vienna, Austria.

Touraïvane, M. Allenbach, M. Mangeas, and C. Bonte (2011). Monitoring the Turbidity Associated with the Dredging in Vavouto Bay in New Caledonia. Paper presented at $19^{\text {th }}$ International Congress on Modelling and Simulation, Perth, Australia, 12-16 December 2011 (http://mssanz.org.au/modsim2011)

Waller, L.A. and C.A. Gotway (2004). Applied Spatial Statistics for Public Health Data. John Wiley \& Sons, 277-280, August $12^{\text {th }}$. 